\title{
VERZEICHNIS DER LIEDERANFÄNGE.
}

[Es sind auch die Anfänge der Einzelstrophen der mehrstrophigen Lieder aufgenommen. Die Zahlen in () bezeichnen die Seiten des Schmellerschen Druckes von 1847.]

Avdientes audiant

Avwe, avwe, daz ich ie wart geborn. . . . . . . (73) 5

Avve, avve mich hiute unde immer we. . . . . . . . (I05) 29

Chramer gip die varwe mier . . . . . . . . . . . (96) 27

Chume, chume geselle min . . . . . . . . . . . . (208) 20

Den sihe ich iemerlichen an . . . . . . . . . . . (105) 29

Der alder werlt ein meister si . . . . . . . . . . (228) 25

Der bremen hohgezit zergat . . . . . . . . . . . . (108) 7

Der cheuer sich selb betriuget . . . . . . . . . . . (I08) 7

Der esel und di nahtigal . . . . . . . . . . . . . . . (108) 8

Der hofwart und der wint . . . . . . . . . . . . (108) 7

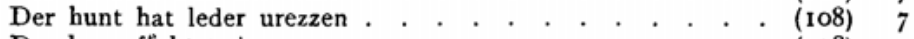

Der lewe fürhtet des mannes . . . . . . . . . . . (108) 7

Der lewe sol auch nimmer lagen . . . . . . . . . . (107) 7

Der starche winder hat uns uerlan . . . . . . . . . (177) II

Der winder zeiget sine chraft . . . . . . . . . . . (214) 22

Die cheuern uliegen unuerdaht . . . . . . . . . . . (I08) 7

Die frossche tůnt in selben schaden . . . . . . . . . (I 48 ) 7

Di wisen chunnen wol uerstan. . . . . . . . . . . (108) 7

Div fliuge ist, wirt der summer heiz . . . . . . . . (107) 7

Div heide grůnet vnd der walt . . . . . . . . . . (182) 13

Div mich singen tůt . . . . . . . . . . . . . . (226) 25

Diu mukke múz sich sere mưn . . . . . . . . (107) 6

Diu nahtigal diche můt . . . . . . . . . . . . . (108) 7

Div werlt frôvt sih uber al . . . . . . . . . . . . (I98) 17

Do er zů der linden chom . . . . . . . . . . . . (216) 24

Deusal misir bescher deuin . . . . . . . . . . . . (233) 26

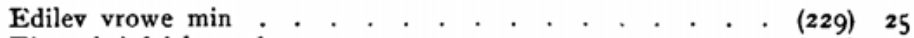

Einen brief ich sande. . . . . . . . . . . . . . (2I2) $2 \mathrm{I}$

Eine wunechliche stat . . . . . . . . . . . . . . (200) 17

Er graif mir an daz wize gewand . . . . . . . . . . (216) 24

Er graif mir anden wizen lip . . . . . . . . . . . (Anm.) 24

Ernam den chocher unde den bogen . . . . . . . . . (Anm.) 24

Er nam mich bi der wizen hant. . . . . . . . . . (216) 24

Er sprach vrowe gewir baz . . . . . . . . . . . . (216) 24

Er wart mir ûf daz bemdelin . . . . . . . . . . . . (Anm.) 24

Es dunchet mich ein tumber $\sin$. . . . . . . . . . (107) 6

Floret silua nobilis . . . . . . . . . . . . . . . (188) 15

Floret silva undique . . . . . . . . . . . (188) 15 
Gienge ein hunt .

Seite

Herzelieb hat manich man

(I07) 6

Hic volucres celi referam . . . . . . . . . . . . . (175) 9

Ia wolde ih an die wisen gan . . . . . . . . . . . (216) 23

Ich pin cheiser ane chrone ............ (188) 15

Ich chume niht von den fuoezzen din . . . . . . . (99) 28

Ich gesach den sumer nie . . . . . . . . . . . . (190) I6

Ich gib ev varwe deu ist guot . . . . . . . . . . . (97) 28

Ich han eine senede not . . . . . . . . . . . . . (207) 19

Ich han gesehen daz mir indem herçen . . . . . . . . (185) 14

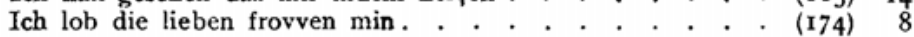

Ich sich den morgen sterne brehen . . . . . . . . . (215) 22

Ich solde eines morgenes gan . . . . . . . . . . $\quad$ (I83) 13

Ich was ein chint so wolgetan . . . . . . . . . . . (216) 23

Ich wil den sumer grüzen . . . . . . . . . . . . (2 II) 2 I

Ich wil truren varen lan.... . . . . . . . . . (203) 21

Th wolde gerne singen. . . . . . . . . . . . . . (209) 17

In liehter varwe stat der walt . . . . . . . . . . . (I79) I 2

Ir roter roservarvver munt . . . . . . . . . . . . (I74) 8

Iz stat ein linde wolgetan . . . . . . . . . . . (2I6) 24

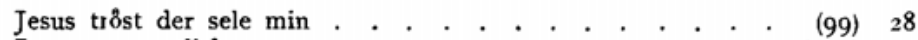

Jesus von gotlicher art. . . . . . . . . . . . . . (107) 30

Lat leben mir daz chindel min . . . . . . . . . . (I05) 29

Manich hunt wol gebaret. . . . . . . (I07) 6

Man minnet nu schatz mere. . . . . . . . . . . . (I08) 8

Man siht uil selten richez hus . . . . . . . . . . . . (108) 7

Mein leben stat in ir gevvalt . . . . . . . . . . . . . (I74) 9

Minne, schatz, groz gewin . . . . . . . . . . . . . (109) 8

Minnet tugentliche man . . . . . . . . . . . . . (96) 27

Min vrowe uenus ist so gît. . . . . . . . . . . . (206) 19

Mir ist ein wip sere . . . . . . . . . . . . (202) 18

Nahtegel, sing einen don. . . . . . . . . . . . . (186) 14

$\mathrm{Nu}$ grvnet auer div heide .. . . . . . . . . . . (204) 18

$\mathrm{Nu}$ lebe ich mir alrest werde . . . . . . . . . . . (72) 5

$\mathrm{Nu}$ sin stolz wnd hovisch. . . . . . . . . . . . . . (188) 15

$\mathrm{Nu}$ sůln wir alle frǒde han . . . . . . . . . . . (18ı) 13

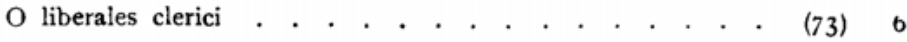

Roter munt wie du dich swachest. . . . . . . . . . . (205) 19

Rusticales clerici . . . . . . . . . . . (73) 6

Sage, daz ih dirs iemmer lone . . . . . . . . . . . (187) 14

$\mathrm{Si}$ ist sch8ner den urowe dido was . . . . . . . . . . (193) 16

Sicut cribratur triticum . . . . . . . . . . . . . . . . . (73) 6

Solde auer ich mit sorgen iemmer leben . . . . . . (202) 18

Fr. Lüers, Die deutschen Lieder der Carmina Burana. 3 
Solde ich noch den tach

So stæte friundin nieman hat . . . . . . . . . . (109) 8

So wol dir meie, wie du scheidest . . . . . . . . . (190) I5

Springerwir den reigen . . . . . . . . . . . . . (I78) I 2

Stetit puella rufa tunica . . . . . . . . . . . (2 I0) 26

Sv̌ziu vrowe min . . . . . . . . . . . . . . . (198) 17

Swa ich waiz den wolues zant . . . . . . . . . . . (107) 7

Swa man den esel chronet . . . . . . . . . . . . . . (I09) 8

Swaz hie gat umbe. . . . . . . . . . . . . . . (203) 18

Swer lieb hat . . . . . . . . . . . . . . . (109) 8

Swer redelicher dinge gert . . . . . . . . . . (I07) 30

Swer schalchait lernt . . . . . . . . . . . . . . (I08) 7

Taugen minne diu ist gůt .

Vns chumet eine liehte sumerzit . . . . . . . . . . (2I4) 22

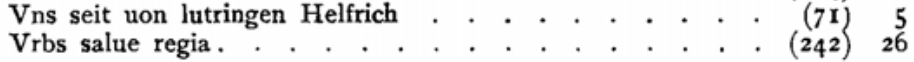

Venit quidam iuvenis . . . . . . . . . . . . . (216) 23

Virgo quedam nobilis . . . . . . . . . . . . . . (216) 23

Von reht iz of in selben gat . . . . . . . . . . (108) 7

Vremede schaidet herzelieb . . . . . . . . . . . . (I09) 8

Vrowe ih pin dir undertan . . . . . . . . . . . . (19I) 16

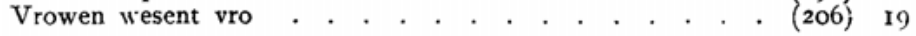

Wart marter ie so iemerlich . . . . . . . . . . . . (105) 29

Waz ist fû́r daz senen gůt . . . . . . . . . . . . . . (227) 25

Wẹre div werlt alle min. . . . . . . . . . . . . (185) 14

Wol dan minneklichev chint . . . . . . . . . . . (97) 27

Wol dir werlt daz du bist . . . . . . . . . . . . (96) 27

Wol ir libe, div so schone . . . . . . . . . . . . (208) 20

Zeniwen vrǒden stat min mût . . . . . . . . . . . (184) 14

Zergangen ist der winder chalt. . . . . . . . . . (180) I 2 